\title{
Influência das Variáveis Biopsicossociais na Qualidade de Vida em Asmáticos
}

\author{
Ana Paula Soares de Matos ${ }^{1}$ \\ Universidade de Coimbra \\ Ana Cláudia Cardoso Machado \\ Rota do Guadiana- Associação de Desenvolvimento Integrado
}

\begin{abstract}
RESUMO - A asma brônquica é uma doença complexa e interfere com a qualidade de vida desses doentes. Pretendemos, com a presente investigação, estudar a relação das variáveis sócio-demográficas (género, idade e grau de instrução), clínicas (gravidade da doença, duração e tipo clínico) e psicológicas (cognições, emoções e comportamentos) com a qualidade de vida do doente asmático. Cinqüenta doentes asmáticos do Departamento de Pneumologia e Imunoalergologia dos Hospitais da Universidade de Coimbra preencheram cinco questionários de auto-resposta que avaliavam as variáveis psicológicas em estudo. Os dados clínicos relativamente à doença foram igualmente recolhidos.Os nossos resultados revelam-nos que a qualidade de vida se relaciona com um conjunto de variáveis que podemos denominar biopsicossociais (e.g., uma menor qualidade de vida relaciona-se com uma maior idade, menor escolaridade, uma atitude mais negativa face à asma, designada "estigma”, cognições disfuncionais e comportamentos/emoções-problema relacionados com a asma). Implicações ao nível do tratamento são apresentadas.
\end{abstract}

Palavras-chave: asma brônquica; variáveis biopsicossociais; qualidade de vida.

\section{Influence of Biopsychosocial Variables in Asthmatics' Quality of Life}

\begin{abstract}
Bronchial asthma is a complex disease and it brings limitations to patients' quality of life. In this research we intend to study the relationship of sociodemographic (gender, age and educational level), medical (severity of the disease, duration and clinical type) and psychological variables (cognitions, emotions and behaviour) with patients' quality of life. Fifty asthmatic patients from the Department of Pulmonary Medicine and Immunoallergic diseases of Coimbra's University Hospitals completed five self-report questionnaires that measured the psychological variables under study. Medical data were also collected. Our results suggest that quality of life is related to a set of variables that can be named as biopsychosocial (e.g., a poor quality of life is related to a higher age, lower level of education, a more negative attitude towards asthma, named "stigma", disfunctional cognitions and behavioural/emotional problems associated to asthma). Implications in relation with the treatment are also presented.
\end{abstract}

Key words: bronchial asthma; bio psychosocial variables; quality of life.

A asma brônquica é uma das doenças crónicas mais comuns e afecta todas as idades. Apesar das dificuldades conhecidas no estabelecimento de uma definição precisa desta doença, dadas as múltiplas utilizações do termo (sintomas, exacerbações, anormalidades das vias aéreas), a asma brônquica é, actualmente, entendida como uma doença inflamatória crónica das vias aéreas que se expressa por episódios recorrentes de pieira, dispneia, aperto torácico e tosse de intensidade variável. Estes sintomas estão normalmente associados a uma obstrução generalizada das vias aéreas, a qual é reversível espontaneamente ou por tratamento (Direcção Geral de Saúde, 2001).

Em Portugal, a prevalência média da asma atinge mais de $11 \%$ da população no grupo etário dos 6-7 anos, $11,8 \%$ no grupo etário dos 13-14 anos e 5,2\% no grupo dos 20-44 anos, estimando-se que existam mais de 600.000 de doentes (Direcção Geral de Saúde, 2002).

1 Endereço: Faculdade de Psicologia e de Ciências da Educação da Universidade de Coimbra, Rua do Colégio Novo, Apartado 6153, Coimbra, Portugal 3001-802.E-mail: apmatos@fpce.uc.pt
Actualmente, esta doença representa um problema social de magnitude considerável que se reflecte em custos financeiros elevados tanto para o doente como para a sociedade. Em termos individuais, pelas suas características clínicas (cursando com agudizações), a doença asmática torna-se um desafio diário à capacidade de adaptação destes doentes, exigindo ajustamentos contínuos no seu funcionamento quotidiano que permitam regular o impacto e o curso da doença.

A avaliação da qualidade de vida dos doentes, em particular dos doentes crónicos, não é uma preocupação recente. Neste contexto, surge como uma avaliação específica dos resultados de saúde e complementar às tradicionais avaliações de "impacto" da doença (e.g., prevalência, mortalidade, índices de hospitalizações e utilização dos recursos médicos).

Qualidade de vida é um conceito subjectivo que se baseia na percepção individual do impacto que têm os acontecimentos e as experiências nas várias esferas de vida dos indivíduos. Está assim relacionada com o nível de bem-estar percebido ou de satisfação pessoal em domínios centrais da vida dos indivíduos (American Thoracic Society, 2004). 
Ao longo dos anos, a literatura científica tem revelado alguma dificuldade na definição e mensuração do conceito, o qual deve ser entendido tendo em conta o enquadramento cultural e social dos indivíduos e os contextos de intervenção (Fayers \& Machin, 2000).

A qualidade de vida relacionada com a saúde não é um conceito unitário, é a componente da qualidade de vida total que é determinada principalmente pelo estado de saúde, constituindo um indicador da percepção que o doente tem do impacto da doença no seu bem-estar e funcionamento diário (Juniper, 2001). Nesta perspectiva, a qualidade de vida reflecte mais do que o estado de saúde, mas sobretudo a maneira como o doente percebe e reage às limitações impostas pela sua doença em três domínios principais: físico, psicológico e social (Silva, Naspitz \& Solé, 2000, Spilker, 1990).

A vivência de uma doença crónica (em especial de começo tardio) envolve um complexo processo de adaptação e traz consigo um conjunto de alterações que repentinamente podem desafiar a visão que o indivíduo tem de si, das suas capacidades e do mundo: a) alterações de papeis pessoais, sociais e profissionais; b) sofrimento físico e psicológico; c) auto-cuidado, por vezes complexo e prolongado; d) interferência ou evidente restrição na realização das actividades diárias (Bishop, 2005).

As recentes abordagens psicológicas e sociais do processo de adaptação à doença permitiram preencher algum vazio conceptual deixado pelas iniciais abordagens causais do modelo médico e psicossomático.

As investigações científicas recentes passaram a utilizar o modelo biopsicossocial (Engel, 1977) que é, por natureza, um modelo sistémico, integrado, quer da saúde, quer da doença, pois considera múltiplos fenómenos interactuantes - físicos, afectivos, cognitivos, comportamentais, interpessoais, sócio-culturais, etc. (Pereira, 2002).

O Modelo do Comportamento de Doença (Mechanic, 1986) surge neste contexto como uma elaboração prática do modelo biopsicossocial de Engel, no qual o papel do significado e dos afectos é central na adopção das estratégias de confronto (McHugh \& Vallis, 1986). A capacidade de adaptação à doença (em especial à doença crónica) passou a ser entendida como um processo individual, multidimensional e altamente subjectivo, orientado pelo conteúdo das significações e interpretações que o indivíduo faz da doença, dos seus sintomas, do tratamento e das estratégias utilizadas no seu confronto.

Da Psicologia da Doença, com fortes raízes cognitivistas (ver Joyce-Moniz \& Barros, 2005), resultou um conjunto de modelos que acentuam a importância dessas representações individuais, das estruturas e processos cognitivos na regulação do comportamento perante a situação de doença. O modelo Auto-regulação das Significações Leigas da Doença, proposto por Howard Leventhal (e.g., Leventhal, Leventhal \& Cameron, 2001; Leventhal, Meyer \& Nerenz, 1980; Leventhal, Nerenz \& Steele, 1984), destaca-se pelo estreito paralelismo que mantém com os pressupostos teóricos dos paradigmas cognitivos (e.g., Beck 1976), tal como têm salientado Joyce-Moniz e Barros (2005): ambos os modelos se fundamentam nas crenças ou significações que a pessoa faz da realidade, apresentam uma hierarquia estrutural e fazem a ligação entre as representações cognitivas, a expressão emocional e os processos de confronto.

Neste contexto, o doente surge como um processador activo de informação que constrói uma representação da sua doença e, em função dela, regula o seu comportamento, assim:

se a significação da doença e do seu confronto é adequada, a pessoa pode controlar as suas emoções e atitudes, respondendo correctamente às situações; mas se a leitura da realidade é má, tudo o resto - reacções emocionais e comportamentos instrumentais - pode ser igualmente mau. (Joyce-Moniz \& Barros, 2005, p. 65).

Na tradição cognitivo-comportamental, o modelo de Beck (1976) não é o único a salientar a importância das representações individuais. Muitos outros proeminentes autores na área da Psicologia têm utilizado esta operacionalização teórica para fundamentar as suas concepções psicopatólogicas e metodológicas (por exemplo, Ellis \& Bernard, 1985; Mahoney, 1991; Young, 1990).

Outros autores como Lazarus (1993; Folkman \& Lazarus, 1991), com os seus estudos sobre o stress e coping, Bandura (1982, 1989), com os seus trabalhos sobre a modelação e auto-eficácia, e Rotter (1966), com as suas pesquisas sobre as atribuições de controlo, têm servido de base a muitos estudos em vários domínios da psicologia, em especial na doença mental e física.

No âmbito da doença asmática esta mudança conceptual é particularmente importante, tendo-se verificado uma crescente incorporação destes conceitos nas suas investigações. Kinsman, Dirks e Jones (1982) introduziram o conceito de psico-manutenção, aproximando-se, assim, das novas tendências conceptuais. A teoria da psico-manutenção debruça-se na forma como as variáveis psicológicas, comportamentais e sociais influenciam o curso da doença, a adesão ao tratamento e a utilização dos recursos médicos, numa perspectiva multidimensinal e dinâmica. As variáveis psicológicas (por exemplo, a ansiedade-traço, as atitudes para com a doença e tratamento, a ansiedade focada nos sintomas), como todas as demais consideradas por este modelo (por exemplo, as sociais), podem ser antecedentes, consequentes ou manter uma relação circular, perpectuando ou agravando o estado físico do doente.

Outros estudos sublinharam a influência de estados emocionais negativos (como a depressão, a presença de sintomatologia ansiosa em períodos de agudização da doença e a negação) no prognóstico da doença, na sua morbilidade, mortalidade e na utilização dos serviços de saúde (Adams, Smith \& Ruffin, 2000; Harrisson, 1998; Miller \& Wood, 1997; Nouwen, Freeston, Labbe \& Boulet, 1999). Num estudo recente realizado por Adams e cols., 2004, verificou-se que a presença de variáveis emocionais e cognitivas negativas, como o distress psicológico/sofrimento e a percepção de um controlo físico pobre, encontram-se significativamente associadas a uma pior qualidade de vida, nomeadamente no domínio do estado de saúde física em doentes asmáticos.

Embora nem sempre sejam concordantes os resultados encontrados pelas diferentes investigações, torna-se cada 
vez mais evidente que os factores psicossociais desempenham um papel importante na asma brônquica, quer como elementos precipitantes das crises, quer na progressão da doença (Presa \& Alonso, 2004).

Considerando assim a importância de uma abordagem pluridisciplinar na compreensão da asma brônquica, pretendemos estudar a relação das variáveis sócio-demográficas (género, idade e grau de instrução), clínicas (nível de gravidade, duração e seu tipo clínico) e psicológicas (atitudes face à doença, cognições disfuncionais, emoções e comportamentos problema associados à asma e sintomatologia pânico-medo) com a qualidade de vida em indivíduos com asma brônquica.

Conceptualizámos um estudo transversal e recorremos a uma pesquisa descritiva de tipo correlacional.

\section{Método}

\section{Sujeitos}

A amostra foi constituída por 50 doentes asmáticos de ambos os sexos, com idades compreendidas entre 16 e 73 anos, assistidos em regime de internamento e consulta externa nos Serviços de Pneumologia e Imunoalergologia dos Hospitais da Universidade de Coimbra, que se prestaram a colaborar, de forma voluntária, no estudo.

A investigação iniciou-se após a aprovação pelas entidades competentes dos respectivos serviços, contando com o apoio por partes das equipas médicas no acesso aos doentes. Neste sentido, para garantir os aspectos éticos envolvidos, foi adoptado o procedimento de consentimento informado, esclarecendo-se previamente todos os participantes acerca dos objectivos e metodologias utilizadas, assegurando-se a confidencialidade da sua participação.

\section{Instrumentos de medida}

Para medir as variáveis sócio-demográficas e clínicas foi construída e utilizada uma Ficha de Informação Clínica, recorrendo-se prioritariamente a entrevistas individualizadas com o doente e o médico responsável pelo seu acompanhamento.

Os sujeitos completaram igualmente cinco questionários de auto-resposta (tipo Likert) que mediam as variáveis em estudo:

- "Respiratory Illness Opinion Survey", de Kinsman, Jones, Matus e Schum (1976), designada por "Escala de Atitudes perante a Doença e o Tratamento". É uma escala de auto-avaliação constituída por 41 itens agrupados em seis sub-escalas: "Optimismo", "Visão negativa do staff clínico", "Especificidade da resposta interna"; "Controlo externo", "Estigma psicológico", "Autoritarismo face à doença e hospitalização". A análise da consistência interna por nós encontrada, revelou a existência de valores $\alpha$ de Cronbach inferiores aos apurados pelos autores, com valores a variarem entre 0,48 ("Visão Negativa do Staff Clínico) e 0,70 ("Estigma Psicológico").

-"Inventário de Cognições Disfuncionais associadas à Asma", de Matos e Machado (1999, citado por Machado, 2003). É um instrumento composto por 37 itens, unidimen- sional, que avalia a frequência de cognições disfuncionais associadas à doença asmática. Apurámos para a nota global um valor de $\alpha$ de Cronbach de 0,93 , o que é indicador de uma boa consistência interna.

- "Revised Asthma Problem Behavior Checklist", de Creer e cols. (1989), designado por "Escala Revista de Comportamentos-Emoções Problema da Asma". É constituída por 76 itens distribuídos por quatro dimensões: "Comportamentos relacionados com a prevenção das crises"; "Comportamentos/Emoções Problema precipitantes das crises"; "Comportamentos/Emoções Problema realizados durante as crises" e "Comportamentos/Emoções Problema consequentes das crises". A escala evidenciou muito boa consistência interna na nota global e nas suas dimensões, com valores a variarem entre 0,94 (nota global) e 0,67 ("Comportamentos relacionados com a prevenção das crises). Os autores também obtiveram um $\alpha$ de Cronbach de 0,94 para a nota global e um valor de teste/re-teste de 0,84 , indicador igualmente de boa estabilidade temporal.

- "Asthma Symptom Checklist (Panic-Fear Symptomatology)”, de Kinsman, Luparello, O’Banion e Spector (1973), designada por "Lista de Sintomas da Asma - Sintomatologia Pânico-Medo". Obtivemos um $\alpha$ de Cronbach de 0,85 para esta dimensão, valor ligeiramente inferior ao encontrado pelos autores $(0,92)$.

- Para avaliar a variável qualidade de vida utilizámos o "Asthma Quality of Life Questionnaire", de Juniper e cols. (1992). O "Questionário de Qualidade de Vida em Doentes Asmáticos" (designação adoptada neste trabalho) é composto por 32 itens que se distribuem por quatro domínios: "Sintomas"; "Limitação de Actividades"; "Emoções" e "Exposição a Estímulos Ambientais". Este instrumento foi traduzido e adaptado para a população portuguesa e aplicado a uma amostra de 165 doentes (em Machado, 2003). O valor obtido para o $\alpha$ de Cronbach foi de 0,959 e a correlação split-half foi de 0,923 , valores indicadores de uma consistência interna elevada.

\section{Tratamento estatístico dos dados}

Os dados foram tratados informaticamente recorrendo ao programa de tratamento estatístico SPSS (Statistical Package for Social Science), na versão 10.0.7 de 1999.

Utilizámos técnicas da Estatística Descritiva e da Estatística Inferencial, nomeadamente: frequências absolutas e relativas; médias aritméticas; desvios-padrão; coeficiente de correlação de Pearson; coeficiente $\alpha$ de Cronbach; regressão linear simples e múltipla; teste $t$ de Student para a diferença de médias entre grupos independentes; análises da variância unifactorial e multifactorial; teste de Tuckey.

\section{Resultados}

As principais características sócio-demográficas da nossa amostra estão representadas na Tabela 1 .

Os doentes apresentavam idades compreendidas entre 16 e $73 \operatorname{anos}(\bar{x}=40,32$ anos; $D P=15,55$ anos), sendo, na sua maioria, do sexo feminino $(80 \%)$. No que respeita ao nível de instrução, verificámos que $38 \%$ dos nossos inquiridos possuía até quatro anos de escolaridade, $20 \%$ possuía entre 
10-12 anos de escolaridade, $16 \%$ apresentava frequência do ensino superior e $14 \%$ detinha entre cinco e nove anos de escolaridade.

Tabela 1. Características sócio-demográficas da amostra.

\begin{tabular}{|c|c|c|}
\hline Variáveis sócio-demográficas & n. ${ }^{\circ}$ & $\%$ \\
\hline \multicolumn{3}{|l|}{ Género } \\
\hline Masculino & 10 & 20,0 \\
\hline Feminino & 40 & 80,0 \\
\hline \multicolumn{3}{|l|}{ Grupo etário } \\
\hline $16-26$ & 12 & 24,0 \\
\hline $26-36$ & 11 & 22,0 \\
\hline $36-46$ & 6 & 12,0 \\
\hline $46-56$ & 12 & 24,0 \\
\hline $56-66$ & 6 & 12,0 \\
\hline $66-76$ & 3 & 6,0 \\
\hline
\end{tabular}

Estado civil

\begin{tabular}{lcc} 
Solteiro(a) & 16 & 32,0 \\
Casado(a) / União de facto & 29 & 58,0 \\
Divorciado(a) / Separado(a) & 2 & 4,0 \\
Viúvo(a) & 3 & 6,0 \\
\hline
\end{tabular}

Habilitações literárias

\begin{tabular}{lcc} 
Sem escolaridade & 1 & 2,0 \\
até 4 anos de escolaridade & 19 & 38,0 \\
$5-9$ anos de escolaridade & 7 & 14,0 \\
$10-12$ anos de escolaridade & 10 & 20,0 \\
Curso Médio & 1 & 2,0 \\
Frequência universitária & 8 & 16,0 \\
Licenciatura & 4 & 8,0 \\
\hline Total & $\mathbf{5 0}$ & $\mathbf{1 0 0 , 0}$ \\
\hline
\end{tabular}

No que respeita às características clínicas da Asma foram analisadas três categorias de variáveis (Tabela 2): Tipo Clínico² (Asma Extrínseca e Intrínseca); Índice de Gravidade da Doença Asmática (conforme classificação proposta pelo projecto - Global Initiative for Asthma (National Institutes of Health, 1995) - implementado pelo National, Heart, Lung and Blood Institute e a Organização Mundial de Saúde); Duração da doença.

Quanto ao tipo clínico, a $86 \%$ dos doentes foi efectuado o diagnóstico de Asma Extrínseca e 14\% sofriam de Asma Intrínseca. Relativamente às características clínicas da asma, 30\% apresentavam Asma Persistente Ligeira, 24\% sofriam de asma ligeira e a mesma percentagem de doentes sofria de Persistente Moderada e 22\% tinham Asma Severa. Quanto

2 Classicamente, e em termos imunológicos, a asma brônquica tem sido classificada em Extrínseca (ou alérgica) e Intrínseca (não alérgica) (Cordeiro, 1995). A primeira ocorre num terreno atópico prévio resultante de uma reacção de hipersensibilidade a uma diversidade de estímulos externos ambientais. Este tipo de Asma caracteriza-se por uma predisposição hereditária e história de atopia. A Asma Intrínseca agrupa todos os casos em que não se detectam mecanismos imunológicos primários, nem história familiar ou individual prévia.
Tabela 2. Características clínicas da amostra.

\begin{tabular}{|c|c|c|}
\hline Variáveis clínicas & n. ${ }^{\circ}$ & $\%$ \\
\hline \multicolumn{3}{|l|}{ Tipo de asma } \\
\hline Intrínseca & 7 & 14,0 \\
\hline Extrínseca & 43 & 86,0 \\
\hline \multicolumn{3}{|l|}{ Índice de gravidade } \\
\hline Ligeira & 12 & 24,0 \\
\hline Persistente ligeira & 15 & 30,0 \\
\hline Persistente moderada & 12 & 24,0 \\
\hline Persistente severa & 11 & 22,0 \\
\hline \multicolumn{3}{|c|}{ Tempo de doença (anos) } \\
\hline$<10$ & 17 & 34,0 \\
\hline $10-20$ & 10 & 20,0 \\
\hline $20-30$ & 8 & 16,0 \\
\hline $30-40$ & 6 & 12,0 \\
\hline $40-50$ & 4 & 8,0 \\
\hline $50-60$ & 5 & 10,0 \\
\hline Total & $\mathbf{5 0}$ & 100,0 \\
\hline
\end{tabular}

à duração da doença, os nossos sujeitos apresentavam uma média de 20,56 anos ( $D P=16,98$ anos).

Estudo da relação entre a Qualidade de Vida e as variáveis Sócio-Demográficas e Clínicas

$\mathrm{O}$ estudo da relação entre a qualidade de vida e a idade revelou a existência de uma correlação predominantemente negativa, sendo significativa na dimensão "limitação de actividades" $(r=-0,37, p=0,009)$. Face aos resultados encontrados pudemos concluir que a qualidade de vida na dimensão "limitação de actividades" tende a diminuir à medida que a idade aumenta.

A comparação da qualidade de vida em função do género dos doentes revelou que não existia qualquer diferença significativa entre os dois grupos $(p>0,05)$. Para compararmos a qualidade de vida em função das habilitações literárias dos doentes considerámos dois grupos, um de doentes com habilitações literárias iguais ou inferiores a nove anos e um outro de doentes com habilitações literárias superiores a nove anos de escolaridade, dado não existir um número representativo de sujeitos de todos os níveis de instrução. Comparando os valores médios obtidos (Tabela 3), pudemos concluir que os doentes com menos escolaridade evidenciavam pior qualidade de vida em todas as dimensões e na nota global.

Por meio da aplicação do teste $t$ de Student pudemos comparar a qualidade de vida dos doentes asmáticos em função do tipo clínico (Extrínseco e Intrínseco). Os resultados apresentados na Tabela 4 permitem-nos constatar que as diferenças observadas são significativas nas dimensões "sintomas", "exposição a estímulos ambientais" e na nota global da qualidade de vida. Pela análise dos valores médios dos dois grupos, podemos concluir que os doentes com diagnóstico de Asma Intrínseca evidenciavam melhor qualidade de vida na nota global e nas dimensões "sintomas" e "exposição a estímulos ambientais" do que os doentes diagnosticados com Asma Extrínseca. 
Tabela 3. Síntese dos resultados da comparação da qualidade de vida conforme as habilitações literárias.

\begin{tabular}{|c|c|c|c|c|c|}
\hline $\begin{array}{c}\text { Dimensões e Nota global da Qualidade de Vida x Habilitações } \\
\text { Literárias }\end{array}$ & $n$ & $\overline{\mathbf{x}}$ & D.P. & $t$ & $p$ \\
\hline \multicolumn{6}{|l|}{ Sintomas } \\
\hline Até nove anos de escolaridade & 26 & 3,43 & 1,36 & 3,701 & 0,001 \\
\hline Mais de nove anos de escolaridade & 23 & 5,03 & 0,96 & & \\
\hline \multicolumn{6}{|l|}{ Limitação de actividades } \\
\hline Até nove anos de escolaridade & 26 & 4,04 & 1,47 & 2,872 & 0,008 \\
\hline Mais de nove anos de escolaridade & 23 & 5,44 & 1,18 & & \\
\hline \multicolumn{6}{|l|}{ Emoções } \\
\hline Até nove anos de escolaridade & 26 & 4,00 & 1,50 & 3,371 & 0,002 \\
\hline Mais de nove anos de escolaridade & 23 & 5,73 & 1,31 & & \\
\hline \multicolumn{6}{|l|}{ Exposição a estímulos ambientais } \\
\hline Até nove anos de escolaridade & 26 & 3,29 & 1,45 & 2,787 & 0,009 \\
\hline Mais de nove anos de escolaridade & 23 & 4,79 & 1,52 & & \\
\hline \multicolumn{6}{|l|}{ Nota Global } \\
\hline Até nove anos de escolaridade & 26 & 3,71 & 1,31 & 3,648 & 0,001 \\
\hline Mais de nove anos de escolaridade & 23 & 5,25 & 0,97 & & \\
\hline
\end{tabular}

Tabela 4. Síntese dos resultados da comparação da qualidade de vida conforme o tipo de asma.

\begin{tabular}{|c|c|c|c|c|c|}
\hline $\begin{array}{c}\text { Dimensões da Qualidade de Vida e Nota Global x Tipo } \\
\text { Clínico }\end{array}$ & $n$ & $\overline{\mathbf{x}}$ & D.P. & $t$ & $p$ \\
\hline Sintomas & & & & & \\
\hline Intrínseca & 7 & 4,96 & 0,99 & 2,309 & 0,025 \\
\hline Extrínseca & 43 & 3,72 & 1,36 & & \\
\hline Limitações de actividade & & & & & \\
\hline Intrínseca & 7 & 5,29 & 1,66 & 1,930 & 0,060 \\
\hline Extrínseca & 43 & 4,13 & 1,44 & & \\
\hline Emoções & & & & & \\
\hline Intrínseca & 7 & 5,66 & 1,76 & 1,488 & 0,143 \\
\hline Extrínseca & 43 & 4,76 & 1,43 & & \\
\hline Exposição a estímulos ambientais & & & & & \\
\hline Intrínseca & 7 & 5,57 & 1,32 & 3,394 & 0,001 \\
\hline Extrínseca & 43 & 3,54 & 1,49 & & \\
\hline Nota Global & & & & & \\
\hline Intrínseca & 7 & 5,26 & 1,30 & 2,438 & 0,019 \\
\hline Extrínseca & 43 & 4,00 & 1,26 & & \\
\hline
\end{tabular}

O teste da análise da variância (ANOVA unifactorial) permitiu-nos comparar a qualidade de vida dos indivíduos em função do índice de gravidade da asma. Os resultados expressos na Tabela 5 permitiu-nos observar diferenças significativas em todas as dimensões e na nota global da qualidade de vida.

Aplicando o teste de Tuckey constatámos que as diferenças significativas ocorreram entre o grupo dos indivíduos que apresentavam Asma Persistente Severa e os indivíduos que evidenciavam Asma Ligeira ou Persistente Ligeira. A leitura dos valores médios encontrados permitiu-nos concluir que os indivíduos com Asma Ligeira ou Persistente Ligeira apresentam melhor qualidade de vida quando comparados com os indivíduos com Asma Persistente Severa. De um modo geral, com o aumento do índice de gravidade da doença tende-se a acompanhar uma diminuição da qualidade de vida dos nossos sujeitos.

Os dados apurados sugerem-nos igualmente a existência de uma correlação negativa significativa entre a duração da doença e a qualidade de vida, na nota global $(r=-0,38$, 
Tabela 5. Síntese dos resultados da comparação da qualidade de vida conforme as categorias do índice de gravidade da asma.

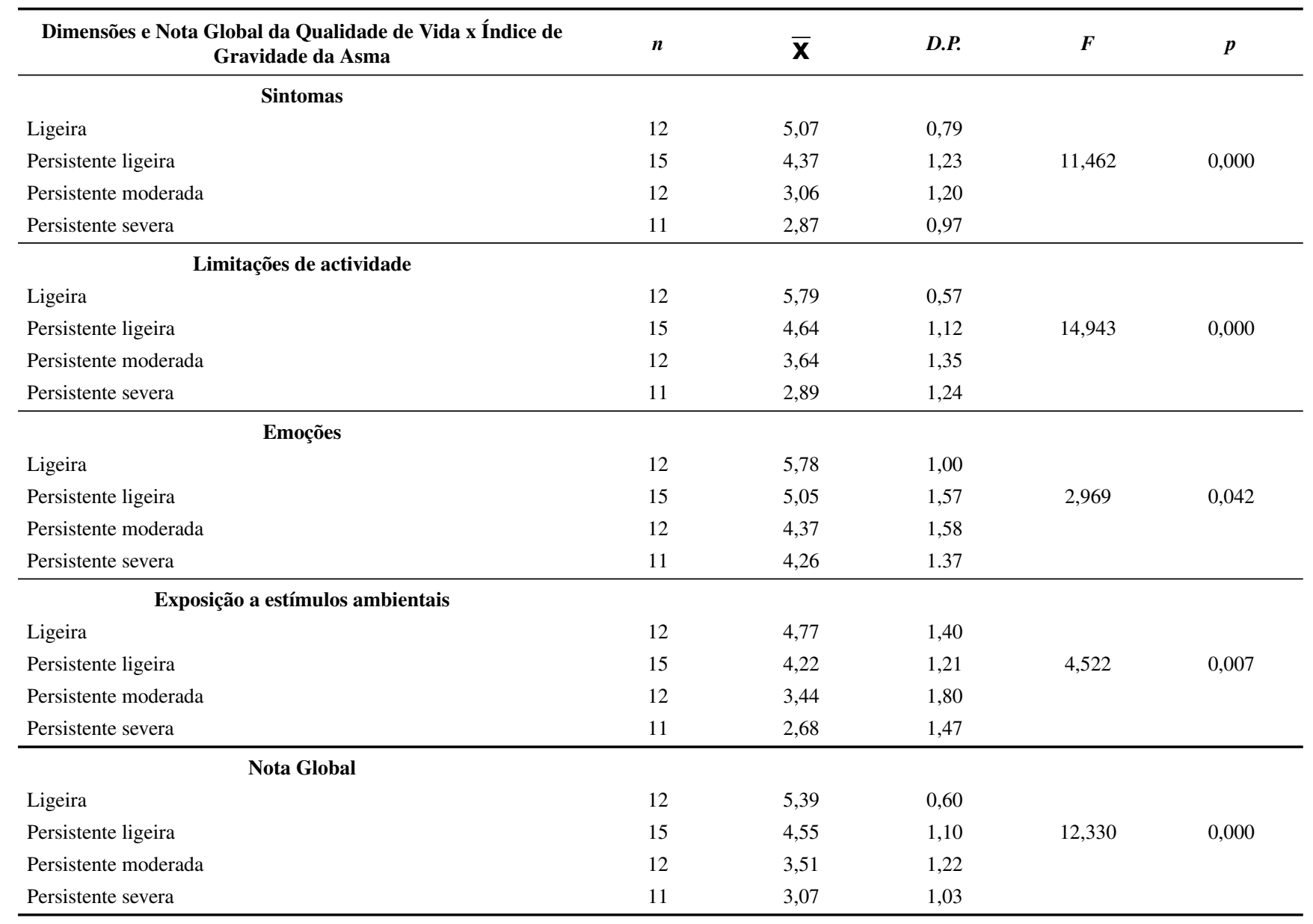

$p=0,007)$, nas dimensões "sintomas" $(r=-0,32, p=0,023)$, "limitação de actividades" ( $r=-0,42, p=0,003)$ e "exposição a estímulos ambientais" ( $r=-0,41, p=0,003)$. Genericamente, com o aumento do tempo de duração da doença tende a verificar-se uma diminuição da qualidade de vida destes doentes.

Estudo de regressão entre a qualidade de vida e as variáveis psicológicas

\section{Qualidade de vida e as atitudes para com a doença e tratamento}

Das análises de regressão efectuadas pudemos verificar que apenas o "Estigma Psicológico" foi seleccionado como preditor de todas as dimensões e nota global da qualidade de vida. Este factor explica $18,2 \%$ da variância na componente "emoções" ( $F=10,713 ; p=0,002), 22,2 \%$ na "exposição a estímulos ambientais" $(F=14,188 ; p=0,000), 22,7 \%$ na dimensão "sintomas" ( $F=14,063 ; p=0,000), 22,9 \%$ na "limitação de actividades" $(F=14,242 ; p=0,000)$ e $27,1 \%$ da variância na nota global $(F=17,809 ; p=0,000)$.

\section{Qualidade de vida e cognições disfuncionais}

O Inventário de Cognições Disfuncionais foi seleccionado como preditor em todas as dimensões e na nota global da qualidade de vida. A percentagem de variância explicada pelo Inventário de Cognições Disfuncionais foi de 9,2\% na dimensão "exposição a estímulos ambientais" ( $F=4,883 ; p=0,032)$, $19 \%$ na dimensão "limitação de actividades" $(F=11,265$; $p=0,002), 26,7 \%$ na nota global $(F=17,440 ; p=0,000), 27,6 \%$ na dimensão "sintomas" e 29,2\% na dimensão "emoções" da qualidade de vida.

\section{Qualidade de vida e comportamentos/emoções problema relacionados com a asma}

Para as relações estudadas encontramos os seguintes resultados: a) o factor 4 ("comportamentos-emoções problema consequentes das crises") e o factor 3 ("comportamentos-emoções problema realizados durante as crises") foram seleccionados como preditores da qualidade de vida ao nível da dimensão "sintomas", apresentando um valor de variância explicada de $24,3 \%(F=15,417 ; p=0,000)$ e $31,0 \%(F=10,573 ; p=0,000)$, respectivamente; b) o factor 3 ("comportamentos-emoções problema realizados durante as crises") e o factor 2 ("comportamentos-emoções problema precipitantes das crises") são preditores da dimensão "limitação de actividades", explicando respectivamente $31,0 \%$ $(F=16,819 ; p=0,000)$ e $41,7 \%(F=21,599 ; p=0,000)$ da sua variância; c) o factor 4 ("comportamentos-emoções problema 
consequentes das crises") foi o termo de predição aceite para a dimensão "emoções" da qualidade de vida, explicando $32,1 \%(F=22,689 ; p=0,000)$ da variância ocorrida; $d)$ o factor 2 ("comportamentos-emoções problema precipitantes das crises") e o factor 3 ("comportamentos-emoções problema realizados durante as crises") foram os dois termos de predição seleccionados para a dimensão "exposição a estímulos ambientais", explicando, respectivamente, 28,6\% ( $F=19,212$; $p=0,000)$ e $35,6 \%(F=12,970 ; p=0,000)$ da sua variância; e) na nota global da qualidade de vida, constatámos que os dois termos de predição aceites foram os "comportamentosemoções problema consequentes das crises" (factor 4) e os "comportamentos-emoções problema realizados durante as crises" (factor 3). A percentagem de variância explicada na nota global pelo factor 4 foi de $32,9 \%(F=23,548 ; p=0,000)$ e pelo factor 3 foi de $40,7 \%(F=16,148 ; p=0,000)$.

\section{Qualidade de vida e sintomatologia pânico-medo}

A "Sintomatologia Pânico-Medo" foi seleccionada como preditora de todas as dimensões e da nota global da qualidade de vida.

A percentagem de variância da qualidade de vida explicada por esta variável foi de 19,2\% na dimensão "exposição a estímulos ambientais" ( $F=11,392 ; p=0,000), 31,6 \%$ na dimensão "sintomas" $(F=22,195 ; p=0,000), 34,1 \%$ na dimensão "limitação de actividades" ( $F=24,802 ; p=0,000), 37,2 \%$ na dimensão "emoções" $(F=28,379 ; p=0,000)$ e $38,6 \%$ na nota global $(F=30,128 ; p=0,000)$.

Em todas as análises efectuadas, a equação de regressão apresentou uma capacidade preditiva significativa e os coeficientes de regressão padronizados apresentaram valores negativos.

Em síntese e face aos resultados apurados pudemos concluir que a qualidade de vida, nas várias dimensões e na nota global, tendia a diminuir nos doentes que apresentavam: a) valores mais elevados de "Estigma Psicológico"; b) índice mais elevado de cognições disfuncionais relacionadas com a doença; c) maior número de emoções e comportamentos problemáticos relacionados com a asma; d) valores mais elevados na variável "pânico-medo".

\section{Discussão}

De um modo geral, os resultados confirmam a associação esperada entre as variáveis sociais, clínicas e psicológicas e os níveis de qualidade de vida dos doentes asmáticos. O presente estudo revelou que os doentes com idade mais avançada apresentavam menor qualidade de vida na dimensão "limitações de actividades". Quanto à relevância do factor idade, esta tem sido salientada por outros autores. Nos indivíduos com asma brônquica de faixas etárias mais avançadas, a asma parece contribuir para a diminuição da sua qualidade de vida (Dyer, Hill, Stockely \& Sinclair, 1999). Verifica-se a ocorrência de um conjunto de complicações físicas e psicológicas associadas (e.g., maior sintomatologia depressiva, maiores limitações das actividades diárias e um estado de saúde pobre) que dificultam o processo de gestão da doença (Enright, Mclelland, Newman, Gottlieb \& Lebowitz, 1999).
De igual modo, verificámos que os doentes com menores habilitações literárias apresentavam menor qualidade de vida na nota global e em cada uma das suas dimensões. Os nossos resultados parecem confirmar as conclusões encontradas por outros estudos que revelam uma relação positiva entre o nível de instrução e a dimensão "percepção do estado de saúde geral" (Regidor \& cols., 1999).

O nível de instrução parece assim dotar os doentes de um conjunto de competências e benefícios sociais que facilitam a compreensão do tipo de doença e a adaptação aos cuidados que esta exige, tais como: a) maior capacidade para o processamento de informação complexa; b) maior capacidade para interagir com as instituições e profissionais de saúde; c) a construção de uma rede de relações mais sustentada e um maior capital cultural; d) a adopção de comportamentos em prol da saúde (inerente ao percurso de socialização); e) aumentar as oportunidades do indivíduo face ao futuro por uma maior rede de assistência, melhor capacidade de planeamento, maior auto-eficácia e capacidade de controlo da doença (Yen \& Moss, 1999).

Por outro lado, também podemos admitir que os níveis inferiores de escolaridade se encontrem, sobretudo, representados nas classes socio-economicamente mais baixas, sendo por isso natural esperar-se que os grupos com menores condições sociais apresentem mais dificuldades de acesso aos cuidados de saúde e possuam menor informação sobre a doença, sendo-lhes consequentemente mais difícil encontrar estratégias eficazes para a gerir.

$\mathrm{Na}$ relação com a qualidade de vida não se encontraram diferenças significativas em relação ao género. $\mathrm{O}$ resultado encontrado contraria as conclusões de alguns estudos que referem que as mulheres evidenciam mais desconforto físico e psicológico perante o processo de doença quando comparadas com os homens e menor qualidade de vida (e.g., Belloch, Perpina, Martinez-Moragon, de Diego \& Martinez Frances, 2003; Wijnhoven, Kriegsman, Snoek, Hesselink \& Haan, 2001, 2003). No entanto, a ausência de relações estatisticamente significativas pode ser, de certo modo, explicada por questões de natureza metodológica, nomeadamente devido às características finais da nossa amostra no qual o género masculino se encontra nitidamente sub-representado. Em futuros estudos, pensamos ser importante incluir um número mais representativo de homens.

Pela análise da relação estabelecida entre as variáveis clínicas e a qualidade de vida, observámos que os indivíduos com asma intrínseca evidenciavam melhor qualidade de vida na nota global e nas dimensões "sintomas" e "exposição a estímulos ambientais" do que os doentes com asma de tipo extrínseco. A este nível não encontrámos bibliografia com a qual fosse possível confrontar estes resultados, todavia baseando-nos nas características clínicas do tipo de asma extrínseca (ou seja, sua natureza reactiva aos alergenos) parece natural que estes doentes apresentem uma manifestação sintomática acentuada e se vejam forçados a limitar as suas exposições a ambientes que comprometam o seu estado de saúde.

Por outro lado, verificámos que a duração da doença diagnosticada se relacionava negativamente com a qualidade de vida. Pensamos que este resultado pode estar relacionado com o facto de os doentes que têm asma há mais tempo serem também os mais idosos. 
No que se refere ao impacto da gravidade da doença na qualidade de vida, verificámos que os indivíduos com asma mais grave (persistente severa) apresentavam pior qualidade de vida do que os doentes com asma menos grave (ligeira ou persistente ligeira). Consistente com a existência de uma relação negativa entre a qualidade de vida e o aumento de gravidade da doença foi também o estudo realizado por Horak e cols. (2005).

Os resultados deste estudo confirmam a associação esperada entre as variáveis psicológicas (cognitivas, emocionais e comportamentais) e o nível de funcionamento destes indivíduos (qualidade de vida). O papel das variáveis psicológicas na percepção de bem-estar tem sido igualmente salientado por outros autores. Nos conhecidos estudos de Denver (Dahlem, Kinsman \& Horton, 1977; Dirks, 1978; Dirks, Kinsman, Horton, Fross \& Jones, 1978; Kinsman, Dahlem, Spector \& Staudenmayer, 1977) verificou-se uma clara relação entre a ansiedade (demasiado alta ou baixa) e o uso dos recursos médicos (tipo do tratamento e taxas de re-hospitalização).

Maes e Schlosser (1987), num estudo efectuado na Holanda com 397 doentes asmáticos dos 16 aos 74 anos, utilizando igualmente a escala RIOS, concluíram que as cognições (por exemplo, o "estigma psicológico") se relacionavam positivamente com as variáveis número de admissões hospitalares e abstenção ao trabalho e que a ansiedade-traço e um estilo de coping centrado nas emoções se relacionavam negativamente com o bem-estar.

Vazquez (2000), numa investigação com 101 doentes asmáticos dos 14 aos 72 anos, verificou que a qualidade de vida apresentava uma relação negativa significativa com muitas variáveis psicológicas relevantes para a evolução clínica da doença: vulnerabilidade percebida, ansiedade/traço, irritabilidade, fadiga, hiperventilação, pânico-medo durante as crises; contudo não apresentava uma relação significativa com os comportamentos de prevenção, comportamentos relacionados com as crises, capacidade de controlo da função respiratória ou qualidade da assistência, tendo concluído que os investigadores devem incluir a análise das variáveis cognitivas, emocionais e comportamentais na avaliação da qualidade de vida destes doentes.

A interacção entre a actividade cognitiva, emoções e comportamentos é uma das características centrais da experiência humana, não podendo ser dissociada dos processos de adaptação à doença. Esta relação de interdependência entre a dimensão biológica e a dimensão psicológica por um lado e entre a cognição-afecto-comportamento por outro tem sido amplamente testada e defendida pelos modelos cognitivo-comportamentais. Este modelo unificador da vivência humana salienta que: a) a capacidade de processar informação e construir significados é central na vivência humana; b) uma função básica do processamento da informação consiste na construção pessoal da realidade; c) todos os seres humanos respondem primariamente à representação cognitiva dos acontecimentos (internos ou externos) e não aos acontecimentos em si; d) o processamento cognitivo dos acontecimentos (internos ou externos) influencia os componentes emocional, comportamental e fisiológico da experiência humana; e) os pensamentos, sentimentos e cognições são vistos como reciprocamente interactivos; f) a actividade cognitiva pode ser monitorizada; g) o disfuncionamento psicológico deve-se fundamentalmente à operação de cognições desadaptadas (Alford \& Beck, 1977; Clark, Beck \& Alford, 1999).

Os nossos resultados realçam esta dialéctica entre os processos físicos e psicológicos e a sua importante influência no nível de funcionamento dos doentes asmáticos. Por outro lado, também não podemos deixar de referir e de aceitar que as variáveis qualidade de vida e as características clínicas da doença (por exemplo, o tipo clinico, gravidade, tempo de duração) poderão afectar o funcionamento psicológico dos indivíduos, estabelecendo-se entre todas as variáveis relações de reciprocidade causal.

O delineamento transversal da investigação reserva-nos, contudo, algumas limitações no completo esclarecimento das relações causais que se estabelecem entre as variáveis. Este facto remete-nos para a necessidade de se efectuarem futuras investigações de caracter longitudinal e em amostras mais alargadas para a maior compreensão deste fenómeno.

Pensamos, no entanto, que os resultados do nosso estudo reforçam a importância de se desenvolverem programas de intervenção psicológica que visem a melhorar a adaptação da pessoa à realidade da doença, promovendo a sua qualidade de vida. De igual modo, sublinha a necessidade de se efectuar uma avaliação biopsicossocial, de múltiplas dimensões, tendo em conta capacidades funcionais, aspectos psicológicos/comportamentais e o funcionamento social destes doentes, o que permitirá desenhar intervenções que melhor se ajustem a cada doente e às suas necessidades.

A ênfase recente colocada numa abordagem pluridisciplinar da doença preconiza adopção de estratégias de educação e de intervenção psicológicas como complemento às abordagens médicas/farmacológicas. Put, van den Bergh, Lemaigre, Demedts e Verleden (2003), num estudo com 23 doentes asmáticos, efectuaram um programa combinado de educação sobre a doença e de intervenção cognitivo-comportamental e verificaram resultados bastante promissores, que se mantinham aos três meses de seguimento: uma diminuição da morbilidade da doença, uma melhoria da qualidade de vida relacionada com a saúde, maior adesão ao tratamento, maior nível de conhecimento sobre a doença e de auto-eficácia para lidar com a mesma, atitudes mais positivas face à doença e menor negatividade.

Outros estudos mostram igualmente a eficácia do tratamento cognitivo- comportamental combinado com a educação sobre a asma. Sommaruga e cols. (1995), avaliaram um programa de mudança cognitiva e comportamental para doentes asmáticos e verificaram que os doentes sujeitos a intervenção combinada apresentavam uma diminuição significativa na variável locus de controlo externo e uma diminuição assinalável dos distúrbios psicofisiológicos (o que reflecte a eficácia do treino de relaxamento e a consequente diminuição da actividade psicofisiológica) e da sintomatologia depressiva. Também observaram uma melhoria dos parâmetros médicos da doença. Por outro lado, a variável "causalidade externa" aumentou significativamente no grupo que apenas beneficiou o plano de educação sobre a doença. Os autores realçam a eficácia das intervenções psicológicas voltadas para a identificação e modificação de 
cognições e emoções desajustadas que possam comprometer a capacidade de percepção e o manejo da doença.

A este nível, os nossos resultados sublinham também a necessidade de se adoptarem estratégias de avaliação e intervenção psicológicas que visem a modificação de cognições e emoções desadaptativas e de padrões comportamentais problemáticos associados à doença asmática por outros mais adaptados e que tragam maior funcionalidade e bem-estar.

O psicólogo deve, deste modo, manter uma relação colaborativa não só com o doente e a família mas também com os profissionais de saúde que o acompanham, sendo por isso pertinente a sua integração em equipas biopsicossociais. Com o doente deve trabalhar, numa primeira fase, no sentido de identificar as cognições e os estados emocionais dominantes que possam ser fonte de sofrimento psicológico. Torna-se por isso fundamental que envolva o doente neste processo de avaliação, que avalie as suas expectativas e que o ajude a aceitar a importância dos aspectos psicológicos, nomeadamente cognitivos, na forma como vê e lida com a sua doença (e.g., a percepção dos sintomas, adesão à terapêutica farmacológica).

É por essa razão fundamental que o psicólogo forneça um racional compreensivo da doença, clarificando as interacções recíprocas entre cognições, afectos, comportamentos, processos biológicos e ambiente. $\mathrm{O}$ terapeuta deve trabalhar colaborativamente com o doente e, por meio da utilização de estratégias cognitivo-comportamentais, intervir em vários aspectos: a) explorar e mudar crenças e processos cognitivos (e.g., pensamento catastrófico, atribuições, auto-afirmações); b) promover estratégias de coping mais adequadas para lidar com os acontecimentos estressantes (e.g., as crises de asma); c) avaliar e trabalhar os factores ambientais e interpessoais que possam estar a contribuir para a manutenção dos sintomas, no qual se inclui a exploração e análise de factos de vida estressantes ou o modelamento e reforço dos sintomas por outras pessoas significativas na esfera de vida do indivíduo.

É, deste modo, necessário que os familiares também participem do processo de intervenção, aprendendo a reconhecer e a reforçar significados e comportamentos funcionais que facilitem o processo de mudança e a experimentação de formas alternativas de adaptação à doença que aumentem o bem-estar do doente.

\section{Referências}

Adams, R. J., Wilson, D. H., Taylor, A. W., Daly, A., Tursan d'Espaignet, E., Dal Grande, E. \& Ruffin, R. E. (2004). Psychological factor and asthma quality of life: a population based study. Thorax, 59(11), 930-935.

Adams, R. J., Smith B. J. \& Ruffin, R. E. (2000). Factors associated with hospital admissions and repeat emergency department visits for adults with asthma Thorax, 55(7), 566-573.

Alford, B. A. \& Beck, A. T. (1977). The integrative power of cognitive therapy. New York: Guilford Press.

American Thoracic Society (2004). Key Concepts: Quality of Life Resource. American Thoracic Society. Retirado em 08/10/2005 do http:/www.atsqol.org./key.asp.

Bandura, A. (1982). Self-efficacy mechanism in human agency. American Psychologist, 35, 122-147.
Bandura, A. (1989). Human agency in social cognitive theory. American Psychologist, 44, 1175-1184.

Beck, A. T. (1976). Cognitive therapy and the emotional disorders. New York: International Universities Press.

Belloch, A., Perpina, M., Martinez-Moragon, E., de Diego, A. \& Martinez Frances, M. (2003). Gender differences in healthrelated quality of life among patients with asthma. Journal of Asthma, 40(8), 945-953.

Bishop, M. (2005). Quality of life and psychosocial adaptation to chronic illness and acquired disability: a conceptual and theoretical synthesis. Rehabilitation Counseling Bulletin, 48(4), 219-231.

Clark, D. A., Beck, A. T. \& Alford, B. A. (1999). Scientific Foundations of Cognitive Theory and Therapy of Depression. New York: Wiley.

Cordeiro, A. J. A. C. (1995). Pneumologia Fundamental. Lisboa: Fundação Calouste Gulbenkien.

Creer, T. L., Wigal, J., Tobin, D., Kotses, H., Snyder, S. \& Winder, J. (1989). The Revised Asthma Problem Behaviour Checklist. Journal of Asthma, 26(1), 17-29.

Dahlem, N. W, Kinsman, R. A. \& Horton, D. J. (1977). Panic-fear in asthma: requests for as-needed medication in relation to pulmonary function measurements. Journal of Allergy and Clinical Immunology, 60(5), 295-300.

Direcção-Geral da Saúde (2001). Manual de Boas Práticas na Asma. Lisboa: Direcção-Geral da Saúde.

Direcção-Geral da Saúde (2002). Ganhos de saúde em Portugal: Ponto de Situação. Relatório do Director-Geral e Alto Comissário da Saúde. Lisboa: Direcção-Geral da Saúde.

Dirks, J. F. (1978). The psycho-maintenance of Bronchial Asthma: A review and preliminary theoretical integration of panicfear research. Report No. 54. Denver: Psychophysiology Research Laboratories, National Jewish Hospital and Research Center.

Dirks, J. F., Kinsman, R. A., Horton, D. J, Fross, K. H. \& Jones, N. F. (1978). Panic-Fear in Asthma: Rehospitalization following intensive long-term treatment: Psychosomatic Medicine, 40(1), 5-13.

Dyer, C. A., Hill, S. L. Stockely, R. A. \& Sinclair, A. J. (1999). Quality of life in elderly subjects with a diagnostic label of asthma from general practice registers. European Respiratory Journal, 14(1), 39-45.

Ellis, A. \& Bernard, M. E. (1985). Clinical applications of rationalemotive therapy. New York: Plenum Publishing Co.

Engel, G. (1977). The need for a new medical model: a challenge for biomedicine. Science, 196, 129-136.

Enright, P. L., Mclelland, R. L., Newman, A. B., Gottlieb, D. J. \& Lebowitz, M. D. (1999). Underdiagnosis and undertreatment of asthma in the elderly. Chest, 116(3), 603-613.

Fayers, P. M. \& Machin, D. (2000). Quality of life: assessment, analysis and interpretation. Chichester: John Wiley and Sons Ltd.

Folkman, S. \& Lazarus, R. S. (1991). Coping and emotion. Em A. Monat \& R. S. Lazarus (Orgs.), Stress and Coping, an anthology ( $3^{\text {rd }}$ ed., pp. 207-227). New York: Columbia University Press.

Harrison, B. D. W. (1998). Psychological aspects of asthma in adults. Thorax, 53(6), 519-525.Horak, E., Sawer, S. M., Roberts, M., Lanigan, A., Carlin, J. B., Olinsky, A. \& Robertson, C. F. (2005). Impact of disease severity on quality of life in adults with asthma. Wien Klin Wochenschr, 117(13-14), 462-467.

Joyce-Moniz, L. \& Barros, L. (2005). Psicologia da doença para cuidados de saúde. Porto: Edições Asa. 
Juniper, E. F. (2001). Using humanistic health outcomes data in asthma. Pharmaco Economics, 19(2), 13-19.

Juniper, E. F., Guyatt, G. H., Epstein, R. S., Ferrie, P. J., Jaeschke, R. \& Hiller, T. K. (1992). Evaluation of impairment of healthrelated quality of life in asthma: development of a questionnaire for use in clinical trials. Thorax, 47(2), 76-83.

Kinsman, R. A., Dahlem, N. W., Spector, S. L. \& Staudenmayer, H. (1977). Observations on subjective symptomatolgy, coping behavior, and medical decisions in asthma. Psychosomatic Medicine, 39, 102-119.

Kinsman, R. A., Dirks, J. F. \& Jones, N. F. (1982). Psychomaintenance of chronic illness: Clinical assessment of personal styles affecting medical management. Em T. Millon, C. Green \& R. Meagher (Orgs.), Handbook of Clinical Health Psychology (pp. 435-465). New York: Plenum Press.

Kinsman, R. A., Luparello, T., O’Banion, K. \& Spector, S. (1973). Multidimensional analysis of the subjective symptomatology of asthma. Psychosomatic Medicine, 35(3), 250-267.

Kinsman, R. A. Jones, N. F., Matus, I. \& Schum, R. A. (1976). Patient variables supporting chronic illness: a scale for measuring attitudes toward respiratory illness and hospitalisation. Journal of Nervous and Mental Disease, 163(3), 159-165.

Lazarus, R. S. (1993). From Psychological stress to the emotions: A history of changing outlooks. Annual Review of Psychology, $44,1-21$.

Leventhal, H., Leventhal, E. \& Cameron, L. (2001). Representations, procedures, and affect in illness self-regulation: a perceptual cognitive model. Em A. Baum, T. Revenson \& J. Singer (Orgs.), Handbook of Health Psychology (pp. 19-47). New York: Earlbaum.

Leventhal, H., Meyer, D. \& Nerenz, D. R. (1980). The commonsense representation of illness danger. Em S. Rachamn (Orgs.), Contributions to Medical Psychology (vol. 2, pp. 7-30). New York: Pergamon Press.

Leventhal H., Nerenz D. R. \& Steele, D. J. (1984). Illness representations and coping with health threats. Em A. Baum, S.E. Taylor \& J. Singer (Orgs.), Handbook of Psychology and Health (pp. 219-252). Hillsdale, New Jersey: Lawrence Earlbaum Associates.

Machado, A. C. (2003). Variáveis Psicológicas na Doença Asmática: Um Estudo à luz do Modelo Cognitivo-Comportamental. Dissertação de Mestrado, Universidade de Coimbra, Coimbra.

Maes, S. \& Schlosser, M. (1987). The Role of Cogniton and Coping in Health Behavior Outcomes of Asthmatic Patients. Current Psychological Research \& Reviews, 6(1), 79-90.

Mahoney, M. J. (1991). Human Change Processes: The Scientific Foundations of Psychoptherapy. New York: Basic Books.

McHugh, S. \& Vallis, T. M. (1986). Illness Behaviour: Operationalization of Biopsychosocial Model. Em S. McHugh \& T. M. Vallis (Orgs.), Illness Behaviour: A Multidisciplinary Model (pp. 1-31). New York and London: Plenum Press.

Mechanic, D. (1986). Illness behaviour: An overview. Em Em S. McHugh \& T. M. Vallis (Orgs.), Ilness Behavior: A Multidisciplinary Model (pp. 101-109). New York and London: Plenum Press.

Miller, B. D. \& Wood, B. L. (1997). Influence of specific emotional states on automatic reactivity and pulmonary function in asthmatic children. Journal of the American Academy of Child \& Adolescent Psychiatry, 36(5), 669-677.
National Institutes of Health (1995). Global Initiative for Asthma: Asthma Management and Prevention. [S.I.]: National Heart, Lung and Blood Institute.

Nouwen, A, Freeston, M. H., Labbe, R. \& Boulet, L. P. (1999). Psychological factors associated with emergency room visits among asthmatic patients. Behaviour Modification, 23, 217-233.

Pereira, M. G. (2002). A Perspectiva Biopsicossocial na Avaliação em Psicologia da Saúde: Modelo Interdependente. Psicologia: Teoria, Investigação e Prática, 7(2), 183-191.

Presa, I. J. \& Alonso, M. A. T. (2004). Revisión: Asma y factores psicosociales. Alergologia e Immunologia Clinica, 19 (3), 101-109.

Put, C., van den Bergh, O., Lemaigre, V., Demedts, M. \& Verleden, G. (2003). Evaluation of an individualised asthma programme directed at behavioural change. European Respiratory Journal, 21, 109-115.

Regidor, E., Barrio, G., de la Fuente, L., Domingo, A, Rodríguez, C. \& Alonso, J. (1999). Association between educational level and health related quality of life in Spanish adults. Journal of Epidemiology and Community Health, 53(2), 75-82.

Rotter, J. (1966). Generalized expectancies for internal versus external control of reinforcements, Psychological Monographs, 90, 1-28.

Silva, M. G., Naspitz, C. K. \& Solé, D. (2000). Qualidade de Vida nas doenças Alérgicas: Por que é importante avaliar? Revista Brasileira de Alergia e Imunopatologia, 23(6), 26-269.

Sommaruga, M., Spanevello, A., Migliori, G. B., Neri, M., Callegari, S. \& Majani, G. (1995). The effects of a cognitive behavioural intervention in asthmatics patients. Monaldi Archives of Chest Disease, 50(5), 398-402.

Spilker, B. (1990). Introduction. Em B. Spilker (Org.), Quality of life assessments in clinical trials (pp. 3-9). NewYork: Taven Press.

Vazquez, M. I. (2000). Relationships between psychological variables relevant to asthma and patients' quality of life. Psychological Reports, 86(1), 31-33.

Wijnhoven, H. A., Kriegsman, D. M., Snoek, F. J., Hesselink, A. E. \& Haan, M. (2001). Differences between men and women in reporting of symptoms during an asthma exacerbation. Annals of Emergency Medicine, 38(2), 123-128.

Wijnhoven, H. A., Kriegsman, D. M., Snoek, F. J., Hesselink, A. E. \& Haan, M., (2003). Gender differences in health-related quality of life among asthma patients. Asthma, 40(2), 189-199.

Yen, I. H. \& Moss, N. (1999). Unbundling education: A critical discussion of what education confers and how it lowers risk for disease and death. Em N. E. Adler, M. Marmot, B. S. McEwen \& J. Stewart (Orgs.), Socioeconomic Status and Health in Industrial Nations: Social, Psychological and Biological Pathways (pp. 350-351). New York: New York Academy of Sciences.

Young, J. (1990). Cognitive Therapy for Personality Disorders: A schema-focused approach. Sarasota: Professional Resource Exchange, Inc.

Recebido em 23.11.2005

Primeira decisão editorial em 28.03.2006

Versão final em 05.08.2006

Aceito em 29.03.2007 